\title{
A breath-taking case of pneumoscrotum
}

\author{
Charles Hesswani $^{* 1}$; Iman Sadri ${ }^{* 1}$; Anne-Marie Canakis ${ }^{2}$; John-Paul Capolicchio ${ }^{1}$ \\ ${ }^{1}$ Division of Urology, Department of Pediatric Surgery, Montreal Children's Hospital, McGill University, \\ Montreal, QC, Canada; ${ }^{2}$ Division Respirology, Department of Pediatrics, Montreal Children's Hospital, McGill \\ University, Montreal, QC, Canada \\ ${ }^{*}$ Equal contributors
}

Cite as: Hesswani C, Sadri I, Canakis A-M, et al. A breath-taking case of pneumoscrotum. Can Urol Assoc J 2022 February 28; Epub ahead of print. http://dx.doi.org/10.5489/cuaj.7830

Published online February 28, 2022

Corresponding author: Dr. J-P Capolicchio, Division of Urology, Department of Pediatric Surgery, Montreal Children's Hospital, McGill University, Montreal, QC, Canada; Jp.Capolicchio.med@ssss.gouv.qc.ca

$* * *$

\section{Introduction}

Pneumoscrotum is a rare physical exam finding that is commonly associated with life-threatening perineal infections or secondary causes from visceral or pulmonary injury. ${ }^{1}$ However, it is usually found in conjunction with multiple other concerning physical exam findings. In this report, we describe a young competitive diver who presented with isolated pneumoscrotum, which eventually was found to be due to a spontaneous tracheal tear

\section{Case report}

A 15-year-old male first presented to the emergency department (ED) of another institution with a one-day history of groin swelling. He described waking up with painful penile/scrotal swelling and crepitus. He proceeded to insert a needle into the area of swelling which released subcutaneous air and lead to a temporary relief of pain. Subsequently, the pain started to increase, and the swelling and crepitus rapidly spread to involve the lower abdominal area and the medial aspects of the thighs. The patient denied experiencing fever, nausea, vomiting, constitutional symptoms or lower urinary tract symptoms There was no history of sick contacts, travel, self-inflicted injury to the genitals or sexual activity. His past medical history was significant for a 1-year history of haematochezia with no findings of 
inflammatory bowel disease after endoscopic studies. He had a remote episode of right testicular torsion-detorsion which was treated conservatively.

On physical examination, significant crepitus and erythema were noted on the scrotum, penile shaft, bilateral medial thighs, and lower abdomen up to the umbilicus. The testicular examination was benign. There were no signs of inguinal lymphadenopathy, trauma, desquamation, or necrosis. Initial laboratory investigations showed normal leukocyte count, electrolytes, erythrocyte sedimentation rate and urinalysis. Urine, wound, and blood cultures were sent, and the patient was started on intravenous broad-spectrum antibiotics and admitted to the hospital. A CT of the abdomen and pelvis demonstrated extensive subcutaneous emphysema predominantly around the scrotum with air pockets extending to the penis, perineum, thighs, and abdominal wall. (Figure 1). However, there was no subcutaneous air present in the thorax or upper aspect of the abdomen (Figure 1). The CT also did not show evidence of abdominal inflammatory changes, free fluid, or abscess.

Over the following days, the patient's clinical and radiological findings improved as his subcutaneous emphysema was gradually resorbed. Antibiotics were discontinued due to negative cultures. He was discharged in a stable condition. Two weeks later, an outpatient MR enterography was normal with no peristalsis abnormalities, fistulas, obstruction, wall thickening or lymphadenopathy.

Seven weeks after discharge, the patient presented to our institution for a second opinion after waking up with left arm/shoulder pain and swelling. Again, the patient noticed air underneath the skin. The swelling progressively extended to the neck and chest area, leading to pleuritic chest pain, orthopnea, dysphagia and vomiting. He had no fever and denied blood in his vomitus. He denied any trauma or recent illness prior to the event. Interestingly, it was discovered that the patient was a competitive diver and had spent around a minute at the bottom of a pool prior to the first episode of subcutaneous emphysema. However, he had not dived prior to the second admission.

The physical exam showed extensive palpable subcutaneous emphysema on the left jaw, neck, arm, and trunk. Genital exam was normal. The chest X-ray showed the presence of pneumomediastinum, pneumopericardium, and significant subcutaneous emphysema. However, no pneumothorax or effusions were appreciated. A flexible laryngoscope performed by the otolaryngology team was normal. The patient was admitted to the floor and placed on IV antibiotics. The patient's status progressed extensively during the hospital stay and lead to almost total body subcutaneous emphysema resulting in brachial plexus compression and pneumoscrotum. An esophagogram demonstrated no evidence of leak. CT of the chest showed extensive pneumomediastinum with emphysema tracking down to the flank area. CT of the neck demonstrated extensive emphysema in multiple planes of the neck, including the retropharyngeal spaces, carotid sheaths and in between the muscles of the floor of the mouth with no obvious point of entry. A discontinuity along the right posterolateral wall of the trachea, measuring around $2 \mathrm{~mm}$ in diameter was identified (Figure 2). A focal tracheal disruption in this area was therefore suspected. A rigid bronchoscopy revealed a cervical/upper thoracic granulation streak at the level of the 5th tracheal ring with slight tracheomalacia. It was suspected that this granulation tissue was likely a healed scar from a 
previous mucosal breach. It was concluded that the subcutaneous emphysema was explained by a tracheal tear and micro-fistula formation which was exacerbated by the sudden pressure changes caused by the patient's diving training. The patient's subcutaneous emphysema progressively resolved with conservative management over the following days and the patient was discharged with close outpatient follow up over 2 years, during which he was advised to avoid diving. During this period, he did not experience a relapse episode of subcutaneous emphysema.

\section{Discussion}

Pneumoscrotum in the pediatric population is a rare finding with a wide range of etiologies. Presentations range from mild and asymptomatic cases to severe with hemodynamic instability. Air may appear in the scrotal wall secondary to intraperitoneal pathologies (gut perforation, entero-scrotal fistulas), infections (necrotizing fasciitis, abscess) or track caudally from the chest wall due to pulmonary causes (barotrauma, thoracic cavity injuries). ${ }^{2}$ Air-leak syndrome is an umbrella term which includes pneumothorax, pulmonary interstitial emphysema, subcutaneous emphysema, pneumomediastinum, pneumopericardium and rarely pneumoperitoneum. The latter also includes pneumoscrotum which is usually associated with concomitant thoracic and abdominal subcutaneous emphysema as the air tracks caudally down the fascial planes. The diagnosis is mainly clinical as crepitus is appreciated on palpation. Performing a CT scan is not mandatory to confirm the diagnosis, but aids in determining the extent and possibly the source of the free air.

Multiple cases were published in the literature describing pneumoscrotum in the pediatric population, mainly secondary to gut perforation or pulmonary barotrauma. Dhir et al. previously reported the case of a 16-day old boy intubated for respiratory distress, who developed extensive subcutaneous emphysema secondary to barotrauma from invasive ventilation. ${ }^{3}$ The emphysema involved the scrotum, chest and abdominal wall. Another case report published in 2007 describes the case of an 18-month-old boy who developed pneumoscrotum, epidural emphysema and pneumomediastinum secondary to an aspiration of a foreign body. ${ }^{4}$ Pneumoscrotum caused by necrotizing fasciitis is a rare condition in the pediatric population but has been described in a 16-year-old boy secondary to perforated appendicitis. $^{5}$

In our particular case, the pneumoscrotum was due to a tracheal tear. Spontaneous tracheal tear is exceedingly rare and has been reported due to coughing and vomiting. ${ }^{6}$ No etiology was found for the tracheal tear in our patient, however it was postulated that the air likely tracked down to the scrotal wall as a result of the patient's diving activities and breathholding. It was theorized that when diving vertically headfirst into deep water, the external immersion pressure exerted on the neck and thoracic cage is higher than that on the scrotum and lower extremities. As a result, any air leak in the subcutaneous tissues would track towards the scrotum. 


\section{Conclusions}

Pneumoscrotum in the paediatric population is a rare finding with multiple etiologies. In this particular population, pneumoscrotum usually presents with concomitant abdominal and chest subcutaneous emphysema. This report describes the first case of an isolated pneumoscrotum due to a pulmonary air leak, more specifically a tracheal tear. 


\section{References}

1. Dagur G, Lee MY, Warren K, et al. Critical Manifestations of Pneumoscrotum. Curr Urol. 2016;9(2):62-66.

2. Khan YA, Akhtar J. Pneumoscrotum: a rare presentation of gastric perforation in a neonate. APSP J Case Rep. 2010;1(2):15.

3. Dhir SK, Kaur A, Kaur G, et al. Massive Air Leak in a Neonate Following Invasive Ventilation. Indian J Pediatr. 2016;83(8):906-907.

4. Dong-Yeon K, Woo-Yeon C, Young-kuk C, et al. Epidural emphysema and pneumoscrotum caused by bronchial foreign body aspiration. Korean Journal of Pediatrics. 2007; 50(8): 785-788.

5. Kauffman JD, O'Brien M, Snyder CW, et al. Acute appendicitis complicated by necrotizing fasciitis in a teenager. Journal of Pediatric Surgery Case Reports. 2018; 37: 77-82.

6. Stevens MS, Mullis TC, Carron JD. Spontaneous tracheal rupture caused by vomiting. Am J Otolaryngol. 2010;31(4):276-278. 


\section{Figures and Tables}

Figure 1. Computed tomography scan views of the chest and pelvis demonstrate subcutaneous air within the thighs and scrotum.

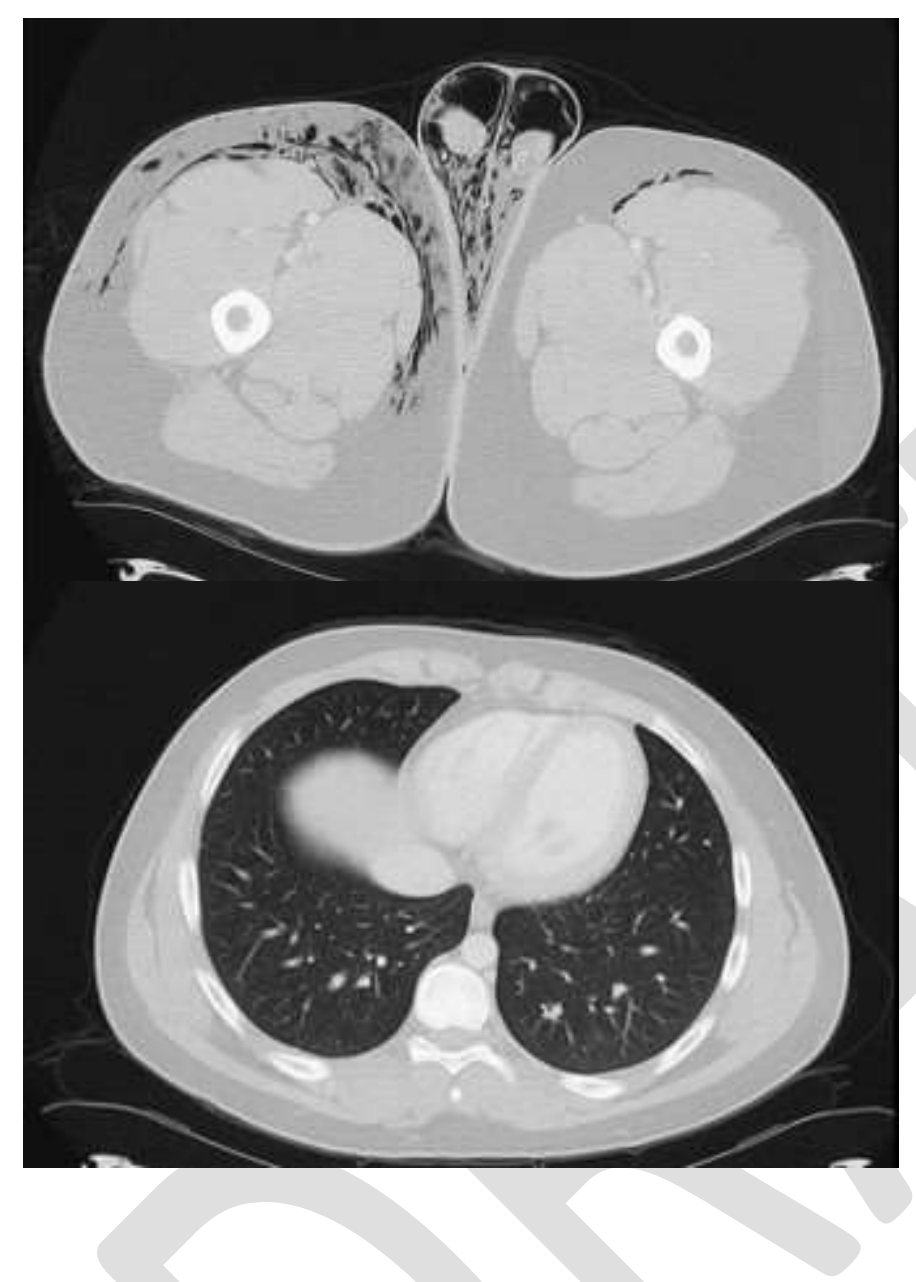

Figure 2. Computed tomography scan of the neck and chest demonstrating the tracheal tear and extensive emphysema.

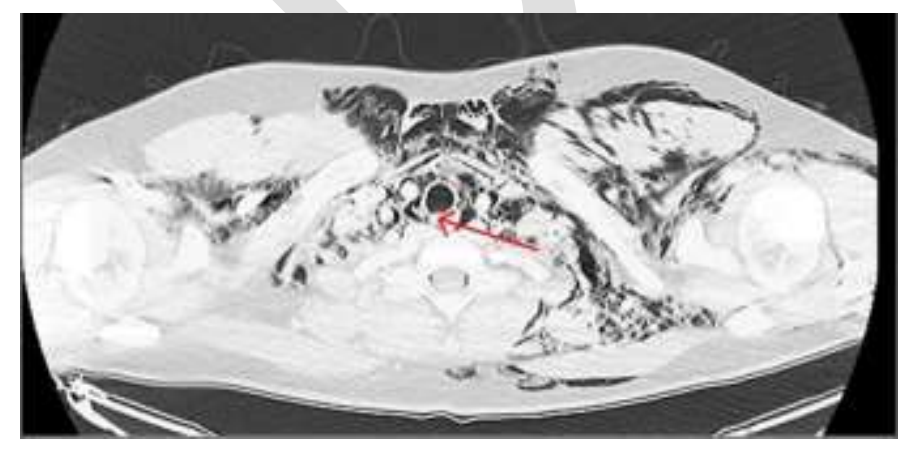

